\title{
FEATURES APPLICATIONS OF THE APPROACHES WHEN CONSTRUCTING EFFICIENT ALGORITHMS DURING THE MODELLING OF SOME INTRACANAL FLOWS
}

\author{
Boris V. Borisov ${ }^{1, \text { a }}$ \\ ${ }^{1}$ National Research Tomsk Polytechnic University, 634050 Tomsk, Russia
}

\begin{abstract}
The methods of increasing the efficiency of the numerical solution of non-stationary problems of gas dynamics for a multicomponent mixture and acceleration the processes of detection of stationary solutions by the establishment method are discussed. Here is considered the relevant algorithms and are shown evaluations of increasing the effectiveness of numerical solutions.
\end{abstract}

Despite the impressive progress in the capabilities of computer technology for many practical applications, there is not least for the important value of the effectiveness of the algorithms. They should reflect as accurately as possible the physical reality and to have the minimum for the one time step for calculate. In this case, these techniques can be widely used in the preliminary evaluation of selectable technical structures or techniques that require multivariate numerical studies. The same time, the economical use of a single calculation requires on-line tracking technology processes. This is especially true for different streams of high-energy working body of the flow path. The high gas velocity causes intensive heat exchange the working fluid of with different structural elements of power aggregate, which ultimately determines the basic parameters of the transient and stationary modes, and thermodynamic properties and viscosity of a multicomponent gas mixture for specifying dynamics of the process.

The system of equations expressing the basic conservation laws are often written in the integral form that is independent of the coordinate system with respect to the control volume $V$, bounded by a closed surface, partially or wholly permeable to gas surface $A$ and impermeable $S$, at which have the heat and mass transfer between gases flow and structural elements:

$$
\begin{aligned}
& \frac{\partial}{\partial t} \int_{V} \rho d V+\int_{A} \rho \cdot N d A=\int_{S} \sum_{j=1}^{L} M_{j} d S, \\
& \frac{\partial}{\partial t} \int_{V} \rho \cdot \boldsymbol{u} d V+\int_{A} \Pi d A+\int_{S} \Pi d S+\int_{S} \rho \cdot \boldsymbol{f} d S=0, \\
& \frac{\partial}{\partial t} \int_{V} E d V+\int_{A}(E+p) \cdot N d A+\int_{S} q d S=\int_{S} \sum_{j=1}^{L} H_{j} \cdot M_{j} d S, \\
& E=p /(\gamma-1)+0.5 \cdot \rho \cdot|\boldsymbol{u}|^{2},
\end{aligned}
$$

\footnotetext{
a Corresponding author: bvborisov@tpu.ru
} 
$t$ - time; $p, \rho, u$ - pressure, density and velocity vector; $N=(\boldsymbol{u}, \boldsymbol{n})-A$ normal to the surface speed is determined by the scalar product of the velocity vector $\boldsymbol{u}$ on the unit vector $\boldsymbol{n}$ the outward normal to the surface; $\quad \boldsymbol{\Pi}=p \cdot \mathbf{n}+\rho \cdot N \cdot \mathrm{u}-$ momentum flux through the surfaces $A$ and $S ; \gamma-$ adiabatic index (ratio of specific heats); $M j, H j$ - the arrival of mass density and enthalpy injected from the surface $\mathrm{S}$ products; $f, q$ - power density of the friction and heat flux.

The real flow is may be accompanied by chemical transformations, the nature of which significantly complicates the mathematical description of the process. However, if the calculation of the equilibrium flow is not an end in itself, its design is in the of practical calculation method in most cases you can restrict a simple and technologically advanced approach "frozen" flow [1]. This approach assumes that the gas "constant" $R$ and the heat capacity are determined by the mass concentration of additive components of the mixture. In [2] proposed two equations describing the change in the local thermodynamic characteristics of a mixture of $R$ and $C p$, replacing any number of equations for an arbitrary number of components in the mixture, which significantly reduces the calculation time:

$$
\begin{aligned}
& \frac{\partial}{\partial t} \int_{V} \rho \cdot R d V+\int_{A} \rho \cdot R \cdot N d A=\int_{S} \sum_{j=1}^{L} R_{j} \cdot M_{j} d S, \\
& \frac{\partial}{\partial t} \int_{V} \rho \cdot C_{p} d V+\int_{A} \rho \cdot C_{p} \cdot N d A=\int_{S} \sum_{j=1}^{L} C_{p j} \cdot M_{j} d S .
\end{aligned}
$$

Index $j$ is used for marking individual properties of the components, $L$ - its quantity in the mixture.

In the majority of cases the practice of determining the hydraulic and thermal interaction of flow with the elements of the setting uses the criterion-experimental dependencies for the coefficients of friction and heat, which in turn is determined by the dimensionless complex that includes a dynamic viscosity $\mu \mathrm{sm}$ mixture of gases. In [3] to calculate $\mu_{s m}$ proposed to use the methodology [2], and particular solutions of the Boltzmann equation. The basic idea, outlined in [3], is that if chemical reactions have little effect on the momentum transfer, then can use the proposed approach to the reaction mixture using a simple approximate expression for calculating the viscosity of the mixture through the molar concentration and molecular weight $M[4,5]$ :

$$
\mu_{s m}=\sum_{i=1}^{N} \mu_{i}\left(1+\sum_{k=1, k \neq i}^{N} \Psi_{i k} \frac{c_{k} M_{i}}{c_{i} M_{k}}\right)^{-1}=\sum_{i=1}^{N} \mu_{i} c_{i}\left(c_{i}+\sum_{k=1, k \neq i}^{N} \Psi_{i k} \frac{c_{k} M_{i}}{M_{k}}\right)^{-1}
$$

$\mu_{\mathrm{i}}$ - Dynamic viscosity coefficient for the $i$-th component, $\Psi$ - viscosity parameters defined by the formulas Enskog-Chapman of the integrals and the diameters of the collision.

With according the approximation of Wilke [4] this dependence reduces to the relation:

$$
\mu_{s m}=\sum_{i=1}^{N} c_{i} \mu_{i}\left(\sum_{j=1}^{N} c_{j} \Psi_{i j}\right)^{-1}
$$

For many number of components it is advisable to apply the simplification Wilke approximation Hëniiga-Tsepperera [5], which reduces it to a simple relation $\Psi_{i j}=\sqrt{M_{i} / M_{j}}=1 / \Psi_{j i}$. After simple transformations, this approach allows for the coefficient of dynamic viscosity of the gas mixture to obtain the relation:

$$
\mu_{\mathrm{cm}}=\sum_{i=1}^{N} c_{i} \mu_{i} \sqrt{R_{i}}\left(\sum_{j=1}^{N} c_{j} \mu_{j} \sqrt{R_{j}}\right)^{-1}
$$


And, taking advantage of the approach used in [2] for the equations (2) to obtain equations for the coefficient of dynamic viscosity of the gas mixture:

$$
\begin{aligned}
& \frac{\partial}{\partial t} \int_{V} \rho \sqrt{R} d V+\int_{V} \rho \sqrt{R} N d A=\int_{S} \sum_{j=1}^{L} \sqrt{R_{j}} M_{j} d S, \\
& \frac{\partial}{\partial t} \int_{V} \rho \sqrt{R} \mu d V+\int_{V} \rho \sqrt{R} \mu N d A=\int_{S} \sum_{j=1}^{L} \sqrt{R_{j}} \mu_{j} M_{j} d S
\end{aligned}
$$

Practice of calculations shows, these equations allows obtaining the coefficient of dynamic viscosity of the gas mixture with an error not exceeding $20 \%$ of the received for more precise, but much more difficult and therefore more costly procedures.

Often the calculation of stationary flow regimes often leads by the method establishing [1]. In this approach, the stationary solution is seen as the limit to which seeks unsteady solution for stationary conditions of flow and mass transfer area of the environment defined by the saturation level of the medium-pressure gas flow in the cavity of the device calculated.

Stationary values of gas-dynamic parameters must correspond with quite trivial relations: the mass flow in the combustion chamber due to $G_{+}$(in many practical cases, there is a functional relationship with the pressure in the form of a power function with an index $<1$ ) is equal to the mass flow of gases through the nozzle $G$ :

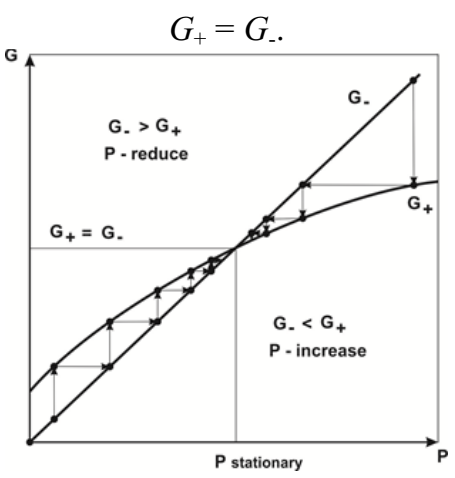

Figure 1. Scheme of the algorithm for finding the pressure that determines the allocation of stationary gasdynamic parameters.

Schematically, this process is shown in Fig. 1. Physically, the process of establishing can be interpreted as the process of removing "superfluous" masses. The analysis shows that the main character of the distribution of flow variables (pressure, speed and temperature) in the canal set very quickly (over a period of time equal to 10 - 30 runs of the sound wave along the length canal) and then slowly seek to the stationary solution.

The "speed" of convergence to this solution is proportional to the velocity of the "removal of excess mass" and seek to "0" with the approach of the pressure to the fixed value of "pressure, determining the arrival of gases through a variety of processes in the cell power plant" and "pressure, determining the expiration of gases from cavities" (Fig. 2).

Let debit and gas consumption as well: $G_{+}=f(p)$ and $G_{-} \approx$ const $p$.

Last ratio in sequence logarithmic, differentiated and recorded in finite differences:

$$
G_{-}=\text {const } \cdot p ; \quad \ln \left(G_{-}\right)=\ln (\text { const })+\ln (p) ; \quad-\frac{\Delta G}{G_{-}}=\frac{\Delta p}{p} ; \Delta p=-p \frac{\Delta G}{G_{-}},
$$

where $\Delta G=G_{+}-G_{-}$.

It has been found experimentally, after some time integration of gas dynamic equations (10 runs of the sound wave along the length of the canal) is achieved quasi-stationary distribution of velocity and 
temperature fields. It is offered that after the integration period to carry out a correction of pressure in each cell and correction of densities on the new values of pressure and temperature already set the levels in accordance with the equation of state.

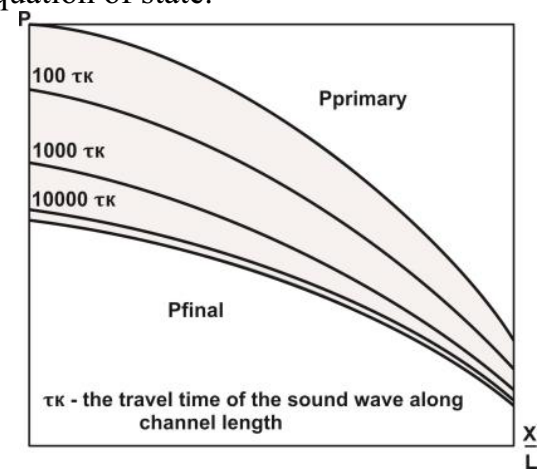

Figure 2. The aspiration process of pressure to the stationary distribution in the model calculations by the method of establishing.

Then the period of integration of gas dynamic equations to the next correction follows. If we write the parameters the before correction with index of "old", after the correction - "kor", and the formula for the $i$ - th cell written in the form $\Delta p_{i}=$ const $p_{i}$, the above-described process of "correcting" the fields of pressure and density for all $i$ can be represented by the following algorithm:

$$
p_{i}^{\text {kor }}=p_{i}^{\text {old }}+\Delta p_{i}=p_{i}^{\text {old }} \cdot\left(1+\Delta p_{i} / p_{i}^{\text {old }}\right) ; p_{i}^{\text {kor }}=p_{i}^{\text {old }} \cdot(1+\text { const }) \text {. }
$$

To maintain the temperature field the field of density is adjusted in proportion to the pressure correction:

$$
\rho_{i}^{\text {kor }}=\rho_{i}^{\text {old }}(1+\text { const })
$$

The calculations show that the use of the algorithm detection of variables in the framework in the method of setting the fields of flow of one-dimensional and two-dimensional approaches reduces the CPU 4 to 100 times, depending on the dimension of the problem. For one-dimensional problems the effect is more obvious. As for spatial calculations, the effect of the acceleration of finding the stationary solutions with this approach to the method of establishing observed, but was less noticeable. The existing experience of the author this paper and proposed approaches for solving various problems of gas dynamic $[1,2,6]$ allows to offer them to build effective methods of numerical simulation of gas-dynamic parameters of real devices to significantly reduce the time spent on carrying out calculations.

\section{References}

1. S.S. Bondarchuk, A.B. Vorozhtsov, A.S. Zhukov, B.V. Borisov, Russ. Phys. J., 57 (12),1796 (2015)

2. S.S. Bondarchuk, A.B. Vorozhtsov, E. A. Kozlov, J. Propul. Power, 11 (4), 593 (1995)

3. J. Hirschfelder., Charles Curtis, R. Byrd, Molecular theory of gases and liquids (New York Wiley, 1964)

4. C.R. Wilke Bondarchuk, J. Chem. Phys., 18 (4), 517 (1950)

5. R.C. Reid, J.M. Prausnitz, B.E. Poling, The properties of gases and liquids (ISBN 0070517991 , MGH, 1987)

6. S.S.;Bondarchuk, B.V. Borisov, A.S. Zhukov, Russ. Phys. J., 55 (9/3), 24 (2012) 